\title{
Hippocampal dysregulation of dopamine system function and the pathophysiology of schizophrenia
}

\author{
Daniel J. Lodge ${ }^{1}$ and Anthony A. Grace ${ }^{2,{ }^{*}}$ \\ ${ }^{1}$ Department of Pharmacology \& Center for Biomedical Neuroscience, University of Texas Health \\ Science Center at San Antonio, San Antonio, TX, USA \\ 2 Departments of Neuroscience, Psychiatry, and Psychology, University of Pittsburgh, Pittsburgh, \\ PA, USA
}

\begin{abstract}
Substantial evidence suggests that psychosis in schizophrenia is associated with a dysregulation of subcortical dopamine system function. Here we examine evidence that this dysregulation is secondary to hyperactivity within hippocampal subfields. Enhanced hippocampal activity has been reported in both preclinical models and in schizophrenia patients. Moreover, this hippocampal hyperactivity is correlated with enhanced dopamine neuron activity and positive symptoms, respectively. Thus, restoration of hippocampal function could provide a more effective therapeutic approach than current therapeutics based on dopamine D2 receptor blockade. Indeed, initial studies demonstrate that allosteric modulation of the $\alpha 5 \mathrm{GABA}_{\mathrm{A}}$ receptor can decrease aberrant dopamine signaling and associated behaviors in a verified rodent model of psychosis.
\end{abstract}

\section{Schizophrenia}

Schizophrenia is a disease with a prevalence of approximately $0.8 \%$ of the population and a lifetime prevalence of about $1 \%$ [1]. It is characterized by three classes of signs and symptoms; positive, negative and cognitive, which are initially reported based on whether the sign or symptom was in addition to (positive), or absent from (negative), normal behavior [2]. Thus, positive symptoms of the disease include hallucinations, delusions and disorganized thoughts, whereas negative signs consist of anhedonia and social withdrawal [2]. Cognitive deficits are also a hallmark of the disease and are typically associated with deficits in attention and executive function [3]. It has been postulated that different symptoms are associated with aberrant activity of distinct brain regions and/or neurotransmitters.

\section{The dopamine hypothesis of schizophrenia}

The neurotransmitter system that has been most commonly associated with schizophrenia is the dopamine system. This 'dopamine hypothesis' is one of the longest standing hypotheses of schizophrenia and is based on multiple observations linking dopamine dysregulation to the pathophysiology of the disease. This includes the finding that enhanced activity within

\footnotetext{
(C) 2011 Elsevier Ltd. All rights reserved.

*Correspondence should be addressed to Dr. Anthony A. Grace, Department of Neuroscience, A210 Langley Hall, University of Pittsburgh, Pittsburgh, PA 15260, USA. GraceAA@ @itt.edu. Tel: +1-412-624-4609. FAX: +1-412-624-9198.

Publisher's Disclaimer: This is a PDF file of an unedited manuscript that has been accepted for publication. As a service to our customers we are providing this early version of the manuscript. The manuscript will undergo copyediting, typesetting, and review of the resulting proof before it is published in its final citable form. Please note that during the production process errors may be discovered which could affect the content, and all legal disclaimers that apply to the journal pertain.
} 
the subcortical dopamine system is associated with the positive symptoms of schizophrenia $[4,5]$ and the fact that all antipsychotic medications are dopamine D2 receptor antagonists $[6,7]$. Moreover, changes in dopamine D2 receptor density are consistently observed in schizophrenia patients (for review see: [8]). Further evidence in support of the dopamine hypothesis has been obtained from imaging studies in human schizophrenia patients that demonstrate significantly increased release of dopamine in the striatum in response to amphetamine administration $[4,9,10]$ with the amplitude of increased dopamine release corresponding to the exacerbation of positive symptoms. More recently, studies have shown that the region of the striatum that shows the largest increase in dopamine release in patients with schizophrenia is related to associative information processing [11]. In addition, dopamine-enhancing drugs (such as amphetamine) precipitate psychotic episodes in normal individuals that are virtually indistinguishable from the acute psychotic episode observed in schizophrenia patients [12]. Taken collectively, these findings lend much evidence and support for the dopamine hypothesis.

However, it is important to note that there has been no substantial pathology reported within the mesolimbic dopamine system of schizophrenia patients. This has led to the suggestion that the pathology might actually be upstream in the systems that regulate the dopamine system, and that the aberrant dopamine transmission and associated symptoms may actually be secondary to dysfunction in cortical and hippocampal regions, which are known to display progressive structural and neurochemical alterations in schizophrenia. Interestingly, this would suggest that the current treatments for schizophrenia (D2 receptor antagonists) might not be the most effective therapeutic approach. Antipsychotic medications, although effective at treating positive symptoms in a subset of patients, do not show a high level of efficacy -- especially across symptom classes -- and exhibit significant side effects [13]. In addition, there is a dramatically high rate (reported at $>74 \%$ ) of patients discontinuing treatment, which demonstrates an urgent need for improved therapies [13]. Here we review recent advances in the understanding of the afferent regions that regulate dopamine system function and provide evidence that targeting afferent regions, specifically the ventral (anterior) hippocampus may be a more effective therapeutic strategy. It should be noted that this hypothesis does not preclude current direct treatments of positive symptoms, but rather, suggests an additional therapeutic strategy for the treatment of schizophrenia.

\section{Altered hippocampal function in schizophrenia}

The hippocampus is a temporal lobe structure essential for the consolidation of long-term memories. Deficits in hippocampal structure and function are a consistent observation in imaging and postmortem studies from schizophrenia patients [14]. Specifically, one of the more constant findings from imaging studies is that patients show an inability to recruit the hippocampus during a task (i.e. memory recall) $[15,16]$. Further evidence for a hippocampal dysfunction came from structural imaging and post mortem analyses that demonstrated consistent anatomical differences, such as a decrease in hippocampal volume, in the brains of schizophrenia patients [17-19]. Thus, several lines of evidence show a decrease in hippocampal function associated with schizophrenia; however, it is important to note that a decrease in function does not necessarily connote a decrease in activity within this structure. Indeed, there is now a significant literature demonstrating that activity within distinct hippocampal subfields is actually increased, at rest, in schizophrenia patients $[14,16,20$ 24]. Specifically, techniques with high spatial resolution have provided evidence for increased regional cerebral blood flow $\left(\mathrm{H}_{2} 0^{15}\right.$-PET: $\left.[22,25]\right)$ and increased regional cerebral blood volume (dynamic susceptibility contrast MRI: [20]) at rest in schizophrenia patients. Importantly, these increases in activity have been directly correlated with clinical measures of psychosis [20,24]. Given that the positive symptoms of the disease are associated with increases in mesolimbic dopamine transmission, one would predict that a 
disruption within the hippocampus could be the source of dopamine system dysregulation in schizophrenia.

\section{Hippocampal regulation of dopamine system function}

The consequence of the purported hippocampal hyperactivity in schizophrenia patients is not entirely known. However, activation of the ventral hippocampus (vHipp) increases dopamine efflux throughout the nucleus accumbens (NAc) of anesthetized rats [26, 27]. Thus, chemical or electrical stimulation of the vHipp produces sustained increases in extrasynaptic dopamine throughout the NAc, a principle target of dopamine neurons [26, 27]. The mechanisms underlying these effects were not immediately apparent because the hippocampus does not directly innervate the dopamine neurons of the ventral tegmental area (VTA). The vHipp could augment dopamine release either via a direct effect on presynaptic dopamine terminals or via the modulation of dopamine neuron activity by means of a polysynaptic pathway. It has now been demonstrated that NMDA activation of the vHipp dramatically increases dopamine neuron activity in the VTA in a dose-dependent manner [28-30]. More specifically, the vHipp modulates dopamine neuron population activity (i.e. the number of neurons tonically active - see Box 1) and this is correlated with an altered dopamine efflux in the NAc [29] and an increased behavioral response to amphetamine [3133]. This increase is dependent on the activation of the NAc by the vHipp because microinfusion of the glutamate antagonist kynurinic acid directly into the NAc blocks vHipp NMDA-induced increases in population activity [28]. Furthermore, the effects of NMDA can be produced by mimicking the effects of NAc excitation-induced increases in GABA receptor activation in the ventral pallidum (VP) [29]. Taken together, these results suggest that the vHipp modulates dopamine neuron population activity via a polysynaptic projection involving a glutamatergic input to the NAc that increases GABAergic activity to the ventral pallidum. This increase in GABA activity decreases tonic VP activity resulting in a disinhibition of dopamine neuron activity in the VTA (Figure 1).

Increases in dopamine neuron population activity not only result in an increase in tonic dopamine efflux in the NAc, but can also regulate the phasic, synaptic dopamine response to stimuli [30]. Specifically, it has been demonstrated that glutamatergic afferents to the VTA can induce burst firing of dopamine neurons in vivo [34]. Furthermore, increases in dopamine neuron burst firing are correlated with increases in dopamine efflux that are localized to the synapse and rapidly curtailed by perisynaptic dopamine transporters [29]. Increases in burst firing are therefore thought to provide the physiologically relevant signal that encodes error prediction or motivational salience. It is important to note that activation of glutamatergic afferents will only induce burst firing in those neurons that are spontaneously active [30]. In neurons that are hyperpolarized by tonic GABAergic inputs from the VP, glutamate will not elicit burst firing due to magnesium blockade of the NMDA receptor. Thus, by regulating the number of dopamine neurons that are spontaneously active, the number of neurons conveying the phasic signal is altered. In this way, the vHipp can dramatically influence dopamine transmission by setting the gain of the system [30]. Disorders of vHipp function will, therefore, result in alteration of this critical gain function.

Such a condition has been modeled using a rat developmental model of schizophrenia. This model involves the administration of a mitotoxin methylazoxymethanol acetate (MAM) to pregnant female rats, specifically on gestational day 17 , which results in a range of anatomical, behavioral and neurochemical deficits that are consistent with those observed in schizophrenia patients (for review see [35]). It should be noted that administration of MAM at earlier time points produces a more widespread histopathology that does not accurately reflect that observed in patients [36]. Consistent with recent human imaging data, MAMtreated rats display baseline hyperactivity of the vHipp [31], and this is associated with a 
significantly higher number of spontaneously active VTA dopamine neurons compared to control rats [31]. This increase in dopamine neuron activity was attributed to hyperactivity within the vHipp because inactivation of the vHipp normalized the augmented dopamine neuron activity, as well as reversed the behavioral hyper-responsivity to amphetamine administration [31]. Therefore, it appears that the hippocampal hyperactivity observed in rodent models as well as in human schizophrenia patients might contribute to the dopamine hyperfunction and associated psychosis (Figure 1). As a result, the gain of the system would be set to maximal (i.e., all of the dopamine neurons are spontaneously firing), causing any stimulus to trigger phasic firing across the entire population of dopamine neurons.

What is the consequence of loss of modulation within the vHipp? Studies suggest that the vHipp is involved specifically in context-dependent processing; i.e., modulating the behavioral response based on the setting. Thus, in settings that are highly activating, such as a dangerous environment or one with potential high rewards (e.g., hunting for food), the vHipp would increase activation of the dopamine system to allow a stimulus to cause a large dopamine response. In contrast, in a benign environment in which stimuli are not likely to have strong survival implications, the vHipp would tone down the dopamine response. However, if the vHipp is pathologically in a constant high-activity state, the dopamine system would always be driven to respond maximally to all stimuli the organism encounters (Figure 2). Such a situation could lead to the attribution of strong behavioral importance to stimuli that otherwise would be safely ignored. This has been termed aberrant salience, and is suggested to potentially underlie delusional or hallucinatory states in schizophrenia [37].

\section{The depolarization block hypothesis of antipsychotic medications}

All current antipsychotic medications have antagonist actions at the dopamine D2 receptor; however, it is evident that antipsychotic drugs achieve their effects via mechanisms that are more complex than the simple blockade of dopamine receptors. Thus, although the maximum rate of improvement occurs early in treatment [38-41], patients also require several weeks of treatment to reach maximum therapeutic responses [38] without evidence of tolerance, as one would expect from simple receptor blockade. Understanding the mechanisms that underlie the therapeutic response to antipsychotic drugs is obviously of fundamental importance both to the understanding of the pathophysiology of schizophrenia and in developing more effective treatments.

Electrophysiological experiments performed in the 1970s provided crucial information regarding the mechanism of action of antipsychotic medications $[42,43]$. Although the acute systemic administration of D2 antagonists to anesthetized rats increases dopamine neuron activity (firing rate and burst firing) potentially via blockade of inhibitory autoreceptors in the VTA, the chronic administration (21 days) of classical antipsychotic drugs produces a dramatic decrease in the number of spontaneously active dopamine neurons [42, 43]. It was demonstrated that this decrease in population activity was attributable to a phenomena known as depolarization block, whereby a substantially depolarized membrane potential results in the inability of the dopamine neurons to generate action potentials [42, 43]. It has been suggested that the induction of depolarization block may be attributable to an interaction between the neuroleptic and anesthetic [44]; however, given that we have previously demonstrated that antipsychotics produce depolarization block in paralyzed rats, this appears unlikely $[42,45]$. Given that paralyzed rats can be easily stressed if not handled appropriately, this likely underlies the differences between these studies. Collectively, studies showing the induction of depolarization block provide important information regarding the clinical efficacy of antipsychotic medications that might explain, in part, the delayed maximal efficacy and lack of tolerance during antipsychotic drug treatment. 
However, recent clinical studies have questioned this hypothesis, largely based on the observation that clinical improvements are not delayed, per se, but rather are gradual, taking weeks to reach maximum effect [38]. The initial electrophysiological studies examining antipsychotic induced depolarization block reported that three weeks of administration is required to produce a decrease in dopamine neuron activity in normal rats. It is important to note, however, that studies in untreated rats might not necessarily reflect results obtained in a pathological condition. Indeed, MAM-treated rats (and presumably schizophrenia patients) display augmented dopamine system function that begins around puberty and persists throughout adulthood. Thus, it is likely that this chronic increase in dopamine transmission results in adaptive changes in dopamine receptor and transporter function. We have recently demonstrated that MAM-treated rats display a significant increase in postsynaptic D2 receptor function throughout both the VTA and NAc [46]. Specifically, the inhibitory effects of the D2 agonist, quinpirole, on dopamine neuron activity are significantly enhanced in MAM-treated rats [46]. Furthermore, the locomotor response to quinpirole is also significantly augmented in this model, suggesting a significant increase in D2 receptor function [46]. Moreover, the initial response of control rats to antipsychotic drugs is a compensatory increase in dopamine neuron population activity; a state that is already present in the MAM-treated rat. Given the significant changes in D2 receptor function and the already-present high level of dopamine neuron firing, we posit that the temporal effects of antipsychotic drug administration might be augmented in MAM-treated rats. Indeed, whereas three weeks of treatment are required to produce depolarization block in control animals, MAM-treated rats display a decrease in population activity upon acute administration of antipsychotic drugs [47]. Therefore, depolarization block is effectively reversing the increase in dopamine neuron population activity in the MAM-treated rat and, unlike control rats, achieves this effect soon after initiation of antipsychotic drug treatment.

\section{The hippocampus as a novel therapeutic target}

Although conventional antipsychotic medications might effectively decrease the positive symptoms of schizophrenia, they are not particularly effective at treating negative signs and cognitive symptoms. This largely reflects the mechanism of action of the D2 antagonists. A decrease in dopamine neuron population activity, due to depolarization block, will reduce the ascribed salience of the dopamine signal; however, it achieves this not by reversing the proposed vHipp overdrive, but instead by inducing an offsetting deficit of depolarization block. Thus, there is still a loss of function whereby the vHipp cannot regulate the gain of the dopamine system (Figure 2). This limits the effectiveness of current antipsychotic treatment,[13]. Antipsychotic medications are not treating the pathology of the disease, but rather produce an abnormal state that offsets the downstream dopamine dysfunction associated with the positive symptoms. Based on the increasing evidence for pathology within the hippocampus underlying the dopamine dysfunction, we posit that restoration of hippocampal function could provide a novel therapeutic approach.

The hyperactivity within hippocampal subfields observed in schizophrenia is likely attributable to a decrease in GABAergic signaling within this region [48, 49]. These GABA deficits, observed postmortem in schizophrenia patient brains, are largely restricted to the class of GABAergic interneurons containing the calcium binding protein parvalbumin [49]. Similarly, we have recently demonstrated a regionally selective decrease in parvalbumin interneuron staining throughout the vHipp of MAM-treated rats [50]. This reduced expression of PV-containing interneurons correlated with a reduction in coordinated neuronal activity during task performance in freely moving rats [50]. A more effective therapeutic strategy might lie in the restoration of GABA interneuron regulation of patterned activity within ventral hippocampal circuits. Interestingly, the use of the GABA modulatory drugs known as benzodiazepines, either alone or as an adjunct to conventional 
antipsychotics, have been extensively studied in schizophrenia patients. Although patient response varies, around one-third of patients improve upon treatment with benzodiazepines [51]. Those who do respond to treatment improve quickly, but the benefits diminish over time. High doses seem to be more beneficial than lower doses, but serious side effects have thus far hampered the feasibility of this treatment. Benzodiazepines act on $\mathrm{GABA}_{\mathrm{A}}$ receptors, which are found throughout the neuraxis. Therefore, it is not surprising that side effects associated with benzodiazepines (sedation, ataxia and cognitive impairment) limit the usefulness of these compounds [51]. By contrast, a benzodiazepine with selectivity for the hippocampus would provide a more effective therapeutic tool.

The $\mathrm{GABA}_{\mathrm{A}}$ receptor is a pentameric structure made from at least eighteen distinct subunits $(\alpha 1-6, \beta 1-3, \gamma 1-3, \delta, \varepsilon, \theta, \rho 1-3)$ [52]. It is the $\alpha$ subunit that provides the allosteric benzodiazepine binding site, and the molecular diversity of this subunit results in distinct functional and anatomical distributions [52]. The $\alpha 1$-containing receptors are the most abundant $(\sim 60 \%)$ and are thought to underlie the sedative properties of benzodiazepines. In contrast, the $\alpha 5$ subunit is much more discretely distributed, with high levels of expression limited to the hippocampus. These receptors are primarily extrasynaptic, localized to the base of the dendritic spines of pyramidal neurons [53]. Thus, the $\alpha 5-\mathrm{GABA}_{\mathrm{A}}$ receptor is positioned to be activated by synaptic GABA as well as 'spillover' from adjacent synapses to provide an inhibitory input to glutamatergic hippocampal projection neurons [53, 54].

Given the specific localization of these receptors to the hippocampus and their purported inhibitory control over hippocampal output, these receptors make an ideal candidate as a potential target to selectively decrease hippocampal activity (Figure 3). Moreover, as this is an allosteric site, drugs acting here would modify GABAergic transmission while preserving the temporal structure necessary for ensembles of neurons to guide behavior [55]. Similarly, the use of allosteric modulators is advantageous from a therapeutic perspective given their decreased potential for toxic effects over conventional agonist drugs. One such compound is SH-053-2'F-R- $\mathrm{CH}_{3}$, a positive allosteric modulator of GABA that displays selectivity and enhanced efficacy at the $\alpha 5$ receptor [56]. The systemic administration of this compound selectively attenuates evoked hippocampal activity in anesthetized rats [57]. Moreover, we have recently demonstrated that this compound can normalize the pathological increase in dopamine neuron population activity in the MAM-model of schizophrenia. In addition, SH-053-2'F-R- $\mathrm{CH}_{3}$, also reduced to associated hyperlocomotor response to psychomotor stimulants in MAM-treated rats [57].

\section{Concluding remarks}

There is increasing evidence from preclinical and human imaging studies suggesting that augmented hippocampal function, secondary to a loss of interneuron function, underlies the dopamine hyperfunction associated with psychosis in schizophrenia. Thus, a restoration of hippocampal function might provide a novel therapeutic target for the treatment of the disease. Indeed, our recent data demonstrate that a positive allosteric modulator of the $\alpha 5 \mathrm{GABA}_{\mathrm{A}}$ receptor can reverse aberrant dopamine system function and, thus, could be effective in treating the dopamine-mediated psychosis. In addition, given that the hippocampus also modulates rhythmic activity that coordinates function across cortical regions implicated in the negative and cognitive symptoms of the disease (such as the prefrontal cortex), restoration of vHipp function may provide a more complete therapeutic profile devoid of the deleterious side effects associated with conventional antipsychotic medications. 


\section{Acknowledgments}

Dr. Grace has the following financial disclosures: Johnson \& Johnson, Lundbeck, Pfizer, GSK, Puretech Ventures, Merck, Takeda, and Dainippon Sumitomo. Dr. Lodge has no conflicts of interest to report.

\section{References}

1. Stilo SA, Murray RM. The epidemiology of schizophrenia: Replacing dogma with knowledge. La epidemiolog Ã-a de la esquizofrenia: Reemplazar el dogma con el conocim. 2010; 12:305-315.

2. Kay SR, et al. The positive and negative syndrome scale (PANSS) for schizophrenia. Schizophrenia Bulletin. 1987; 13:261-276. [PubMed: 3616518]

3. Heinrichs RW, Zakzanis KK. Neurocognitive deficit in schizophrenia: A quantitative review of the evidence. Neuropsychology. 1998; 12:426-445. [PubMed: 9673998]

4. Abi-Dargham A. Do we still believe in the dopamine hypothesis? New data bring new evidence. The international journal of neuropsychopharmacology/official scientific journal of the Collegium Internationale Neuropsychopharmacologicum (CINP). 2004; 7(Suppl 1):S1-5. [PubMed: 14972078]

5. Laruelle M, Abi-Dargham A. Dopamine as the wind of psychotic fire: new evidence from brain imaging studies. Journal of Psychopharmacology. 1999; 13:358-371. [PubMed: 10667612]

6. Creese I, et al. Dopamine receptors and average clinical doses. Science. 1976; 194:546. [PubMed: 17783671]

7. Seeman P, et al. Brain receptors for antipsychotic drugs and dopamine: direct binding assays. Proceedings of the National Academy of Sciences of the United States of America. 1975; 72:43764380. [PubMed: 1060115]

8. Seeman P, et al. Psychosis pathways converge via D2High dopamine receptors. Synapse. 2006; 60:319-346. [PubMed: 16786561]

9. Abi-Dargham A, et al. Increased baseline occupancy of D2 receptors by dopamine in schizophrenia. Proceedings of the National Academy of Science. 2000; 97:8104-8109.

10. Laruelle M, et al. Single photon emission computerized tomography imaging of amphetamineinduced dopamine release in drug-free schizophrenic subjects. Proceedings of the National Academy of Science. 1996; 93:9235-9240.

11. Kegeles LS, et al. Increased synaptic dopamine function in associative regions of the striatum in schizophrenia. Archives of general psychiatry. 2010; 67:231-239. [PubMed: 20194823]

12. Janowsky DS, et al. Provocation of schizophrenic symptoms by intravenous administration of methylphenidate. Archives of general psychiatry. 1973; 28:185-191. [PubMed: 4630714]

13. Lieberman JA, et al. Effectiveness of antipsychotic drugs in patients with chronic schizophrenia. New England Journal of Medicine. 2005; 353:1209-1223. [PubMed: 16172203]

14. Tamminga CA, et al. The hippocampal formation in schizophrenia. American Journal of Psychiatry. 2010; 167:1178-1193. [PubMed: 20810471]

15. Weiss AP, et al. Impaired hippocampal recruitment during normal modulation of memory performance in schizophrenia. Biol Psychiatry. 2003; 53:48-55. [PubMed: 12513944]

16. Heckers $S$, et al. Impaired recruitment of the hippocampus during conscious recollection in schizophrenia. Nature neuroscience. 1998; 1:318-323.

17. Harrison PJ. The neuropathology of schizophrenia. A critical review of the data and their interpretation. Brain. 1999; 122 ( Pt 4):593-624. [PubMed: 10219775]

18. Heckers S, Konradi C. Hippocampal neurons in schizophrenia. Journal of neural transmission. 2002; 109:891-905. [PubMed: 12111476]

19. Nelson MD, et al. Hippocampal volume reduction in schizophrenia as assessed by magnetic resonance imaging: A meta-analytic study. Archives of general psychiatry. 1998; 55:433-440. [PubMed: 9596046]

20. Schobel SA, et al. Differential targeting of the CA1 subfield of the hippocampal formation by schizophrenia and related psychotic disorders. Archives of general psychiatry. 2009; 66:938-946. [PubMed: 19736350] 
21. Malaspina D, et al. SPECT study of visual fixation in schizophrenia and comparison subjects. Biol Psychiatry. 1999; 46:89-93. [PubMed: 10394477]

22. Medoff DR, et al. Probing the human hippocampus using rCBF: contrasts in schizophrenia. Hippocampus. 2001; 11:543-550. [PubMed: 11732707]

23. Lewandowski, NM., et al. Isolating hippocampal subregions most vulnerable to schizophrenia. Annual Society for Neuroscience Meeting Program number: 443.1; 2005.

24. Molina V, et al. Anatomical and functional cerebral variables associated with basal symptoms but not risperidone response in minimally treated schizophrenia. Psychiatry Research - Neuroimaging. 2003; 124:163-175.

25. Tamminga CA, Holcomb HH. Phenotype of schizophrenia: a review and formulation. Mol Psychiatry. 2005; 10:27-39. [PubMed: 15340352]

26. Blaha CD, et al. Stimulation of the ventral subiculum of the hippocampus evokes glutamate receptor-mediated changes in dopamine efflux in the rat nucleus accumbens. European Journal of Neuroscience. 1997; 9:902-911. [PubMed: 9182943]

27. Legault M, Wise RA. Injections of N-methyl-D-aspartate into the ventral hippocampus increase extracellular dopamine in the ventral tegmental area and nucleus accumbens. Synapse. 1999; 31:241-249. [PubMed: 10051104]

28. Floresco SB, et al. Glutamatergic afferents from the hippocampus to the nucleus accumbens regulate activity of ventral tegmental area dopamine neurons. J Neurosci. 2001; 21:4915-4922. [PubMed: 11425919]

29. Floresco SB, et al. Afferent modulation of dopamine neuron firing differentially regulates tonic and phasic dopamine transmission. Nature neuroscience. 2003; 6:968-973.

30. Lodge DJ, Grace AA. The hippocampus modulates dopamine neuron responsivity by regulating the intensity of phasic neuron activation. Neuropsychopharmacology. 2006; 31:1356-1361. [PubMed: 16319915]

31. Lodge DJ, Grace AA. Aberrant hippocampal activity underlies the dopamine dysregulation in an animal model of schizophrenia. J Neurosci. 2007; 27:11424 - 11430. [PubMed: 17942737]

32. Lodge DJ, Grace AA. Amphetamine activation of hippocampal drive of mesolimbic dopamine neurons: a mechanism of behavioral sensitization. J Neurosci. 2008; 28:7876-7882. [PubMed: 18667619]

33. White IM, et al. Amphetamine-induced hyperlocomotion in rats: Hippocampal modulation of the nucleus accumbens. Hippocampus. 2006; 16:596-603. [PubMed: 16763995]

34. Grace AA, Bunney BS. The control of firing pattern in nigral dopamine neurons: burst firing. J Neurosci. 1984; 4:2877-2890. [PubMed: 6150071]

35. Lodge DJ, Grace AA. Gestational methylazoxymethanol acetate administration: A developmental disruption model of schizophrenia. Behav Brain Res. 2009; 204:306-312. [PubMed: 19716984]

36. Moore $\mathrm{H}$, et al. A neurobehavioral systems analysis of adult rats exposed to methylazoxymethanol acetate on E17: implications for the neuropathology of schizophrenia. Biol Psychiatry. 2006; 60:253-264. [PubMed: 16581031]

37. Kapur S. Psychosis as a state of aberrant salience: A framework linking biology, phenomenology, and pharmacology in schizophrenia. American Journal of Psychiatry. 2003; 160:13-23. [PubMed: 12505794]

38. Agid O, et al. Delayed-Onset Hypothesis of Antipsychotic Action: A Hypothesis Tested and Rejected. Archives of general psychiatry. 2003; 60:1228-1235. [PubMed: 14662555]

39. Kapur S, et al. Evidence for onset of antipsychotic effects within the first 24 hours of treatment. American Journal of Psychiatry. 2005; 162:939-946. [PubMed: 15863796]

40. Leucht $S$, et al. Early-onset hypothesis of antipsychotic drug action: A hypothesis tested, confirmed and extended. Biol Psychiatry. 2005; 57:1543-1549. [PubMed: 15953491]

41. Li M, et al. Time course of the antipsychotic effect and the underlying behavioral mechanisms. Neuropsychopharmacology. 2007; 32:263-272. [PubMed: 16738541]

42. Bunney BS, Grace AA. Acute and chronic haloperidol treatment: comparison of effects on nigral dopaminergic cell activity. Life sciences. 1978; 23:1715-1727. [PubMed: 31529] 
43. Grace AA. The depolarization block hypothesis of neuroleptic action: implications for the etiology and treatment of schizophrenia. Journal of neural transmission. 1992; 36:91-131. [PubMed: 1356143]

44. Melis M, et al. Haloperidol does not produce dopamine cell depolarization-block in paralyzed, unanesthetized rats. Brain research. 1998; 783:127-132. [PubMed: 9479061]

45. Chiodo LA, Bunney BS. Possible mechanisms by which repeated clozapine administration differentially affects the activity of two subpopulations of midbrain dopamine neurons. Journal of Neuroscience. 1985; 5:2539-2544. [PubMed: 2863337]

46. Perez, SM.; Lodge, DJ. Increased dopamine receptor sensitivity in an animal model of schizophrenia. Preceedings of the Society for Neuroscience; 2010. p. 62.22

47. Valenti, O., et al. Antipsychotic drugs elicit a rapid depolarization inactivation of VTA DA neuron firing in rat model of schizophrenia via the nucleus accumbens-ventral pallidal pathway. Neuroscience Meeting Planner; 2009. p. 646.10

48. Benes FM, et al. Regulation of the GABA cell phenotype in hippocampus of schizophrenics and bipolars. Proceedings of the National Academy of Sciences of the United States of America. 2007; 104:10164-10169. [PubMed: 17553960]

49. Lewis DA, et al. Cortical inhibitory neurons and schizophrenia. Nature Reviews Neuroscience. $2005 ; 6: 312-324$.

50. Lodge DJ, et al. A loss of parvalbumin-containing interneurons is associated with diminished oscillatory activity in an animal model of schizophrenia. The Journal of neuroscience: the official journal of the Society for Neuroscience. 2009; 29:2344-2354. [PubMed: 19244511]

51. Wolkowitz OM, Pickar D. Benzodiazepines in the treatment of schizophrenia: A review and reappraisal. American Journal of Psychiatry. 1991; 148:714-726. [PubMed: 1674645]

52. Rudolph U, et al. GABAA receptor subtypes: Dissecting their pharmacological functions. Trends in Pharmacological Sciences. 2001; 22:188-194. [PubMed: 11282419]

53. Brunig I, et al. Intact sorting, targeting, and clustering of ?-aminobutyric acid A receptor subtypes in hippocampal neurons in vitro. Journal of Comparative Neurology. 2002; 443:43-55. [PubMed: 11793346]

54. Collinson N, et al. Enhanced learning and memory and altered GABAergic synaptic transmission in mice lacking the 25 subunit of the GABAA receptor. Journal of Neuroscience. 2002; 22:55725580. [PubMed: 12097508]

55. Buzsaki G. Functions for interneuronal nets in the hippocampus. Canadian journal of physiology and pharmacology. 1997; 75:508-515. [PubMed: 9250385]

56. Savic MM, et al. Novel positive allosteric modulators of GABAA receptors: Do subtle differences in activity at [alpha]1 plus [alpha] 5 versus [alpha]2 plus [alpha] 3 subunits account for dissimilarities in behavioral effects in rats? Progress in Neuro-Psychopharmacology and Biological Psychiatry. 2010; 34:376-386. [PubMed: 20074611]

57. Gill, K., et al. Hyperactivation of the dopamine system in the MAM model of schizophrenia is reversed by $25 \mathrm{GABAAR}$ agonist treatment. Preceedings of the Society for Neuroscience; 2010. p. 766.4

58. Grace AA, Bunney BS. Nigral dopamine neurons: intracellular recording and identification with Ldopa injection and histofluorescence. Science. 1980; 210:654-656. [PubMed: 7433992]

59. Grace AA, Bunney BS. Intracellular and extracellular electrophysiology of nigral dopaminergic neurons--1. Identification and characterization. Neuroscience. 1983; 10:301-315. [PubMed: 6633863]

60. Grace A, Onn S. Morphology and electrophysiological properties of immunocytochemically identified rat dopamine neurons recorded in vitro. J Neurosci. 1989; 9:3463-3481. [PubMed: 2795134]

61. Grace AA, Bunney BS. Opposing effects of striatonigral feedback pathways on midbrain dopamine cell activity. Brain Res. 1985; 333:271-284. [PubMed: 2986775]

62. Grace AA, Bunney BS. The control of firing pattern in nigral dopamine neurons: single spike firing. J Neurosci. 1984; 4:2866-2876. [PubMed: 6150070]

63. Schultz W. Predictive reward signal of dopamine neurons. Journal of neurophysiology. 1998; 80:1-27. [PubMed: 9658025] 
64. Grace AA, et al. Regulation of firing of dopaminergic neurons and control of goal-directed behaviors. Trends in Neurosciences. 2007; 30:220-227. [PubMed: 17400299] 


\section{BOX 1}

Dopamine Neuron Activity States

Dopamine neurons in the mesencephalon can be routinely identified in vivo and distinguished from non-dopaminergic neurons by standard electrophysiological criteria $[58,59]$. These neurons typically display a bi-phasic (positive/negative), long duration action potentials that, when measured by extracellular techniques, are greater than $2 \mathrm{~ms}$ in duration $[58,59]$. Dopamine neurons recorded in vivo display three distinct patterns of activity. The first is a 'silent' mode whereby the membrane potential is significantly hyperpolarized and the dopamine neuron is not spontaneously active [58, 59]. Given that dopamine neurons are autonomous pacemakers in vitro [60], this activity state is likely attributable to afferent-mediated inhibition. Non-firing dopamine neurons recorded intracellularly in vivo are consistently bombarded by GABAergic inputs, likely originating from the ventral pallidum [61]. The second activity state is an irregular, single spike firing pattern that is generated intrinsically by pacemaker potentials and regulated by local and remote afferent activity [62]. The third activity pattern consistently observed in dopamine neurons are periods of high frequency activity known as burst firing. High frequency bursts of activity are considered the functionally relevant signal sent to postsynaptic targets to indicate reward or to encode an error prediction signal [63]. 


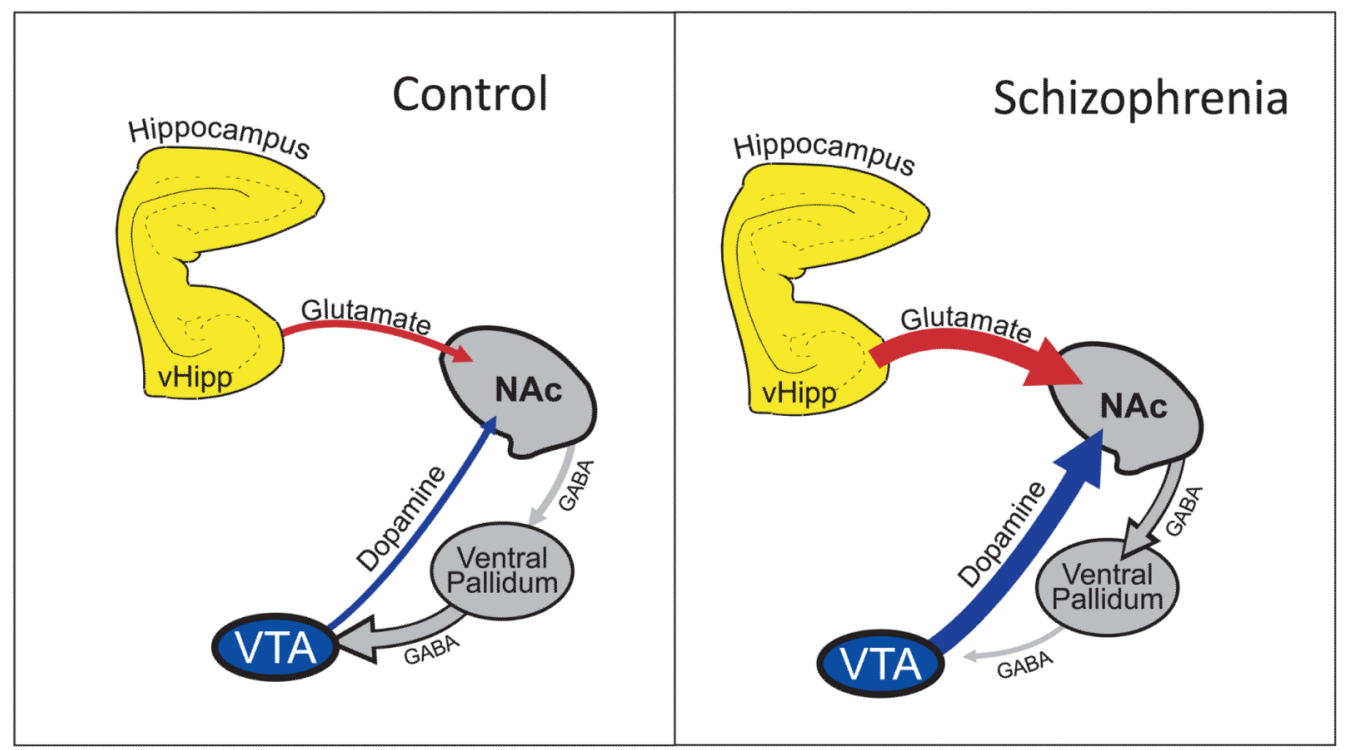

Figure 1.

The ventral hippocampus (vHipp) regulates dopamine neuron activity via a polysynaptic projection. Thus, the vHipp excites neurons in the nucleus accumbens that, in turn, inhibit ventral pallidal (VP) activity. Given that the VP provides an inhibitory tone to the dopamine neurons of the VTA, activation of the vHipp will result in an increase dopamine neuron activity. In schizophrenia, hippocampal activity is pathologically enhanced which leads to an enduring increase in dopamine system function. Adapted from [64]. 


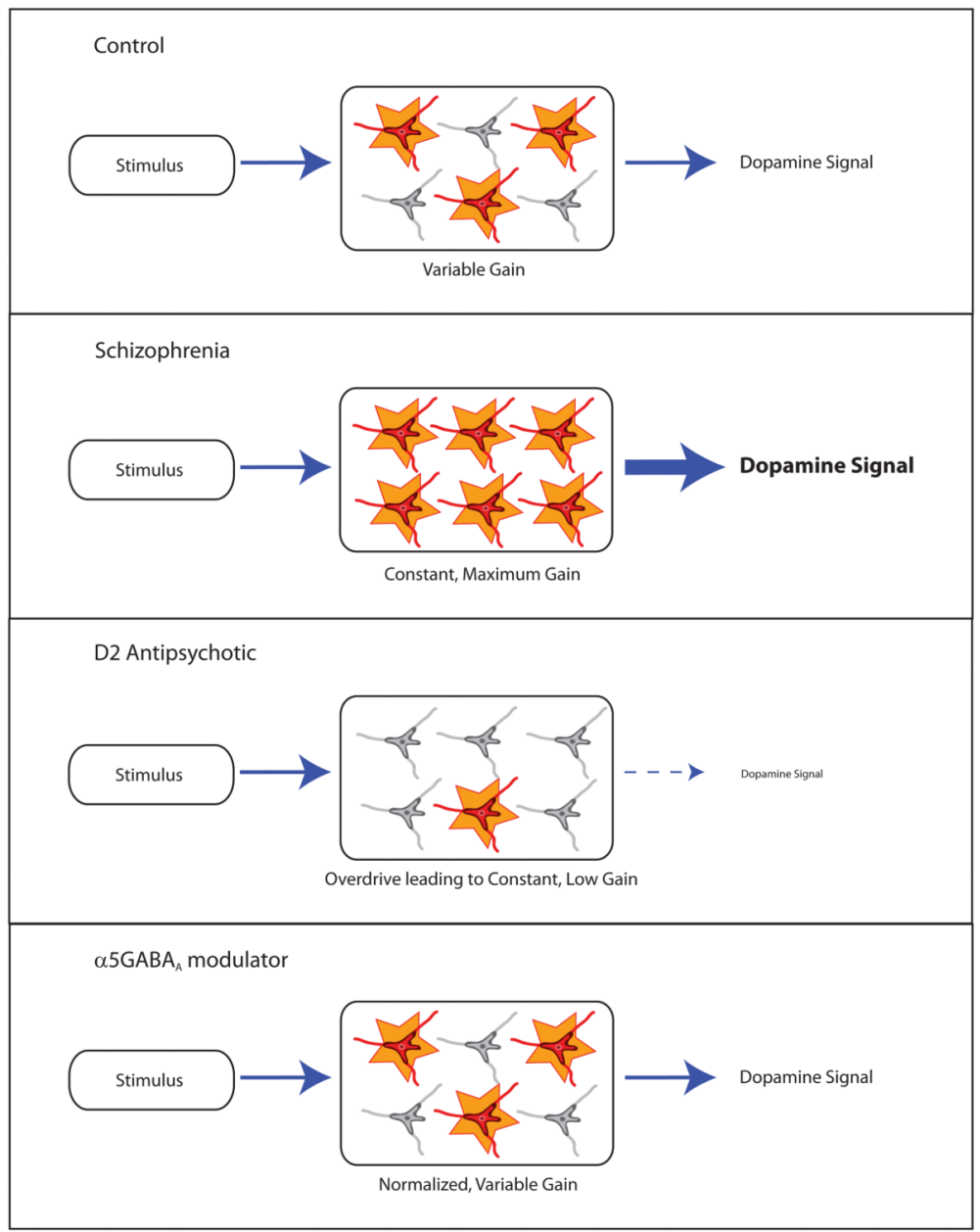

Figure 2.

Under normal (non-pathological) conditions, the number of dopamine neurons spontaneously active can be modulated to permit an appropriate behavioral response based on the environmental setting. In schizophrenia, the dopamine neuron population activity is pathologically in a constant high-activity state and, as such, all stimuli the organism encounters will lead to maximal dopamine output, resulting in the attribution of strong behavioral importance to stimuli, even to stimuli that should be safely ignored. Conventional antipsychotics decrease dopamine neuron activity via depolarization block; this would decrease the salience of all encountered stimuli but fails to restore the flexibility of the system. Novel therapeutics aimed at normalizing hippocampal function, i.e. allosteric modulators of the $\alpha 5 \mathrm{GABA}_{\mathrm{A}}$ receptor, restores normal dopamine function by acting upstream at the proposed site of pathology. 


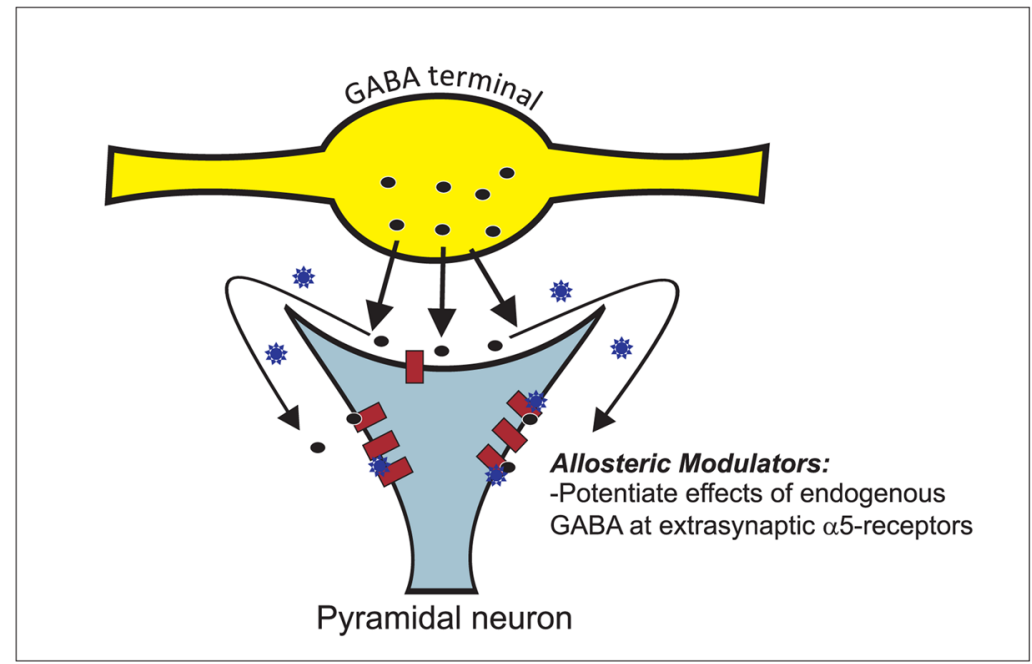

Figure 3.

The alpha 5 subunit is discretely distributed with high levels of expression limited to the hippocampus. These receptors are primarily extrasynaptic, localized to the base of the dendritic spines of pyramidal neurons. Thus, the $\alpha 5-\mathrm{GABA}_{\mathrm{A}}$ receptor is positioned to be activated by synaptic GABA (dark circles) as well as 'spillover' from adjacent synapses to provide an inhibitory input to glutamatergic hippocampal projection neurons. SH-053-2' F$\mathrm{R}-\mathrm{CH}_{3}$ (blue stars) binds to an allosteric, benzodiazepine site of the $\alpha 5-\mathrm{GABA}_{\mathrm{A}}$ receptor, and augments the endogenous inhibition of the pyramidal neuron. 